\title{
Trichophyton erinacei in European hedgehogs admitted to wildlife rehabilitation centres: results of a prospective epidemiological survey in Ile-de-France region, France
}

\author{
LE BARZIC, Cécile ${ }^{1}$, DENAES, Chloé1 ${ }^{\text {, ARNÉ, Pascal }}{ }^{1,2}$, ANGEBAULT, Cécile², BOTTEREL, Françoise ${ }^{2}$, GUILLOT, Jacques $^{2}$, \\ RISCO-CASTILLO, Veronica ${ }^{1,2 *}$ \\ ${ }^{1}$ Centre d'accueil de la Faune Sauvage (CEDAF), Ecole Nationale Vétérinaire d'Alfort, Maisons-Alfort, 94700, France \\ ${ }^{2}$ Dynamyc research group EA 7380, Ecole Nationale Vétérinaire d'Alfort, UPEC, Maisons-Alfort, 94700, France.
}

\section{EnVA UPEC}

\section{Summary}

There is an uprising number of hedgehogs (Erinaceus europaeus) admitted every year to wildlife rehabilitation centres in Western Europe. These animals are frequently found infected by ectoparasites (ticks and mites) as well as the dermatophyte species Trichophyton erinacei. The objective of the present study was to determine the presence of $T$. erinacei in hedgehogs admitted to a wildlife rehabilitation centre from Ile-de-France region, France and to assess the risk of cross contamination in order to adapt control measures.

\section{Methods}

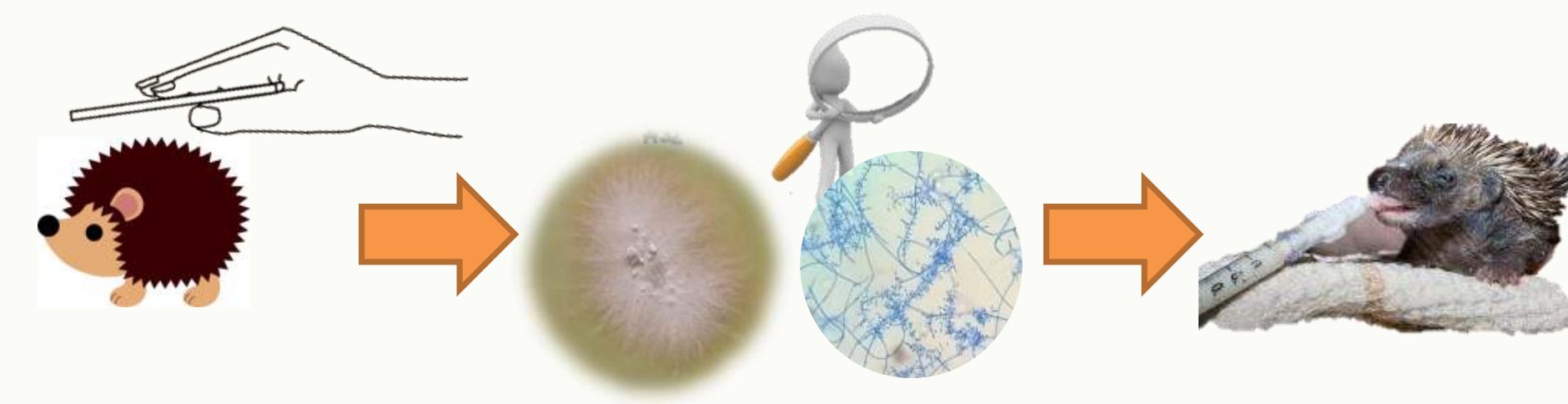

- Collected epidemiological data : origin, weight and sex

- Culture on Sabouraud dextrose agar plates at $32^{\circ} \mathrm{C}$ for 10 days.

- Enilconazole treatment (every 3 days, 4 times) followed by itraconazole (5-10 mg/kg SID for 1 week, 3 times and 1 week after every treatment) if laboratory diagnosis was positive.

\section{Results}

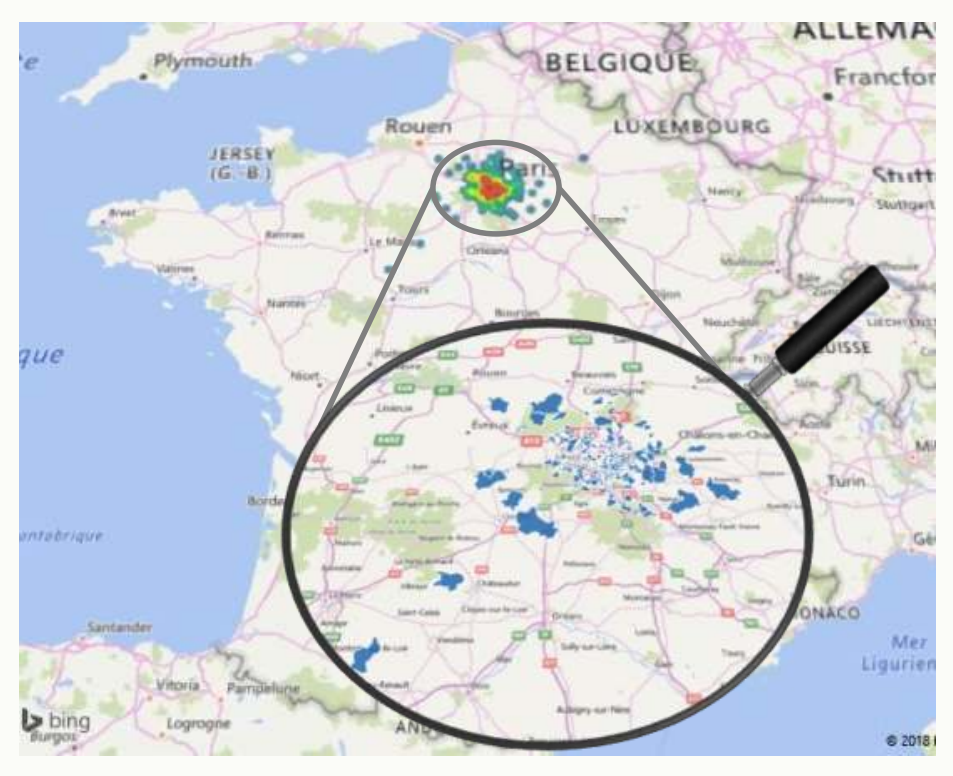

Figure 1. Origin of hedgehogs admitted to CEDAF

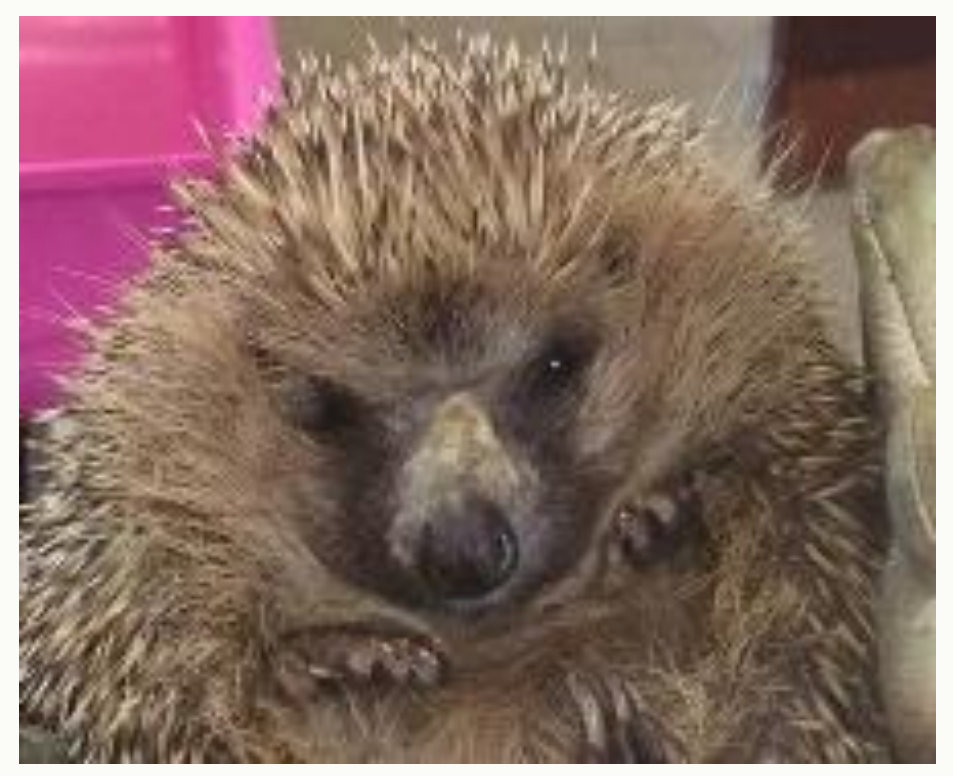

Figure 2. Classical aspect of ringworm lesions on the nose of a hedgehog infected by T. erinacei.

The study included 412 wild hedgehogs (2016) from unweaned hoglets to adults, weighing between 10 and 1711 gr. T. erinacei positive cultures were obtained from 96 hedgehogs $(22.8 \%)$ at arrival. Nannizzia gypsea was detected in two animals (confirmed by MALDI-TOF). Only $10 \%$ of positives showed skin lesions. Dermatophyte detection at admission seemed to be more frequent in low-weighed animals $\left(\chi^{2}, p=0.0673\right)$. T. erinacei was sometimes detected for up to 3 consecutive times despite antifungal treatment. Eleven hedgehogs $(2.6 \%)$ were not infected at their arrival but were later contaminated at least once.

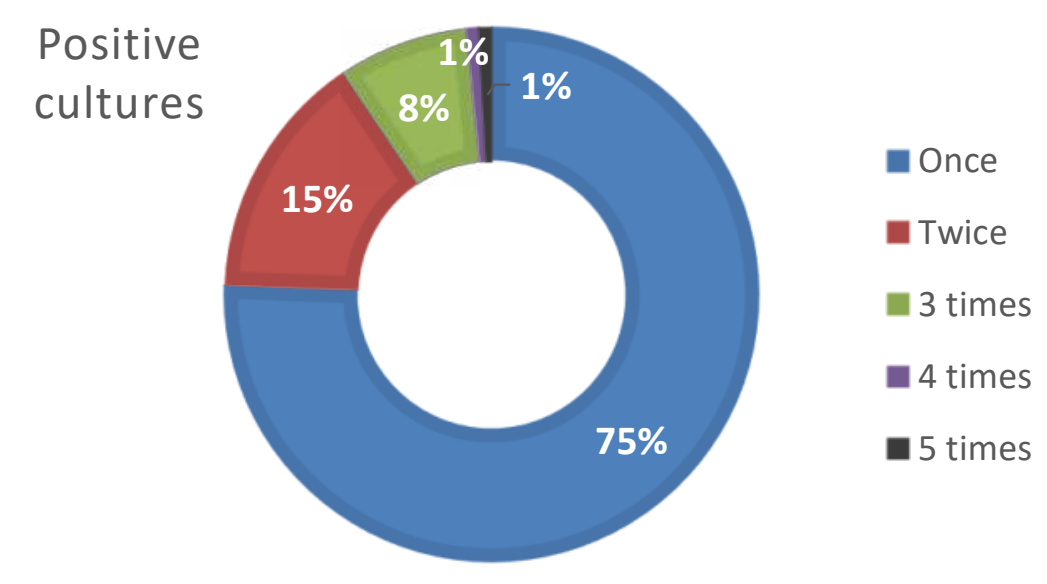

Dermatophyte detection by season

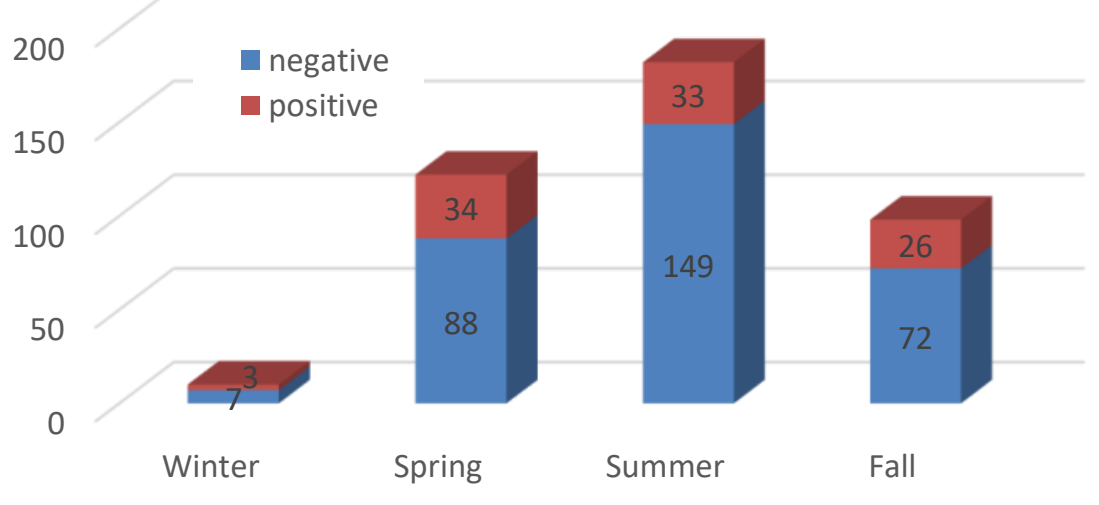

Dermatophyte detection by age

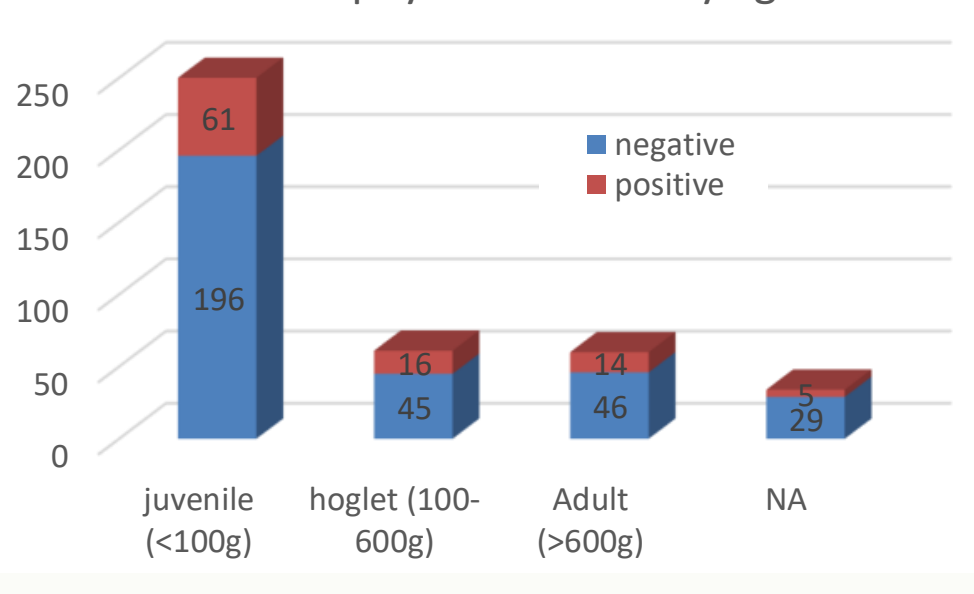

Clinical lesions vs. laboratory detection

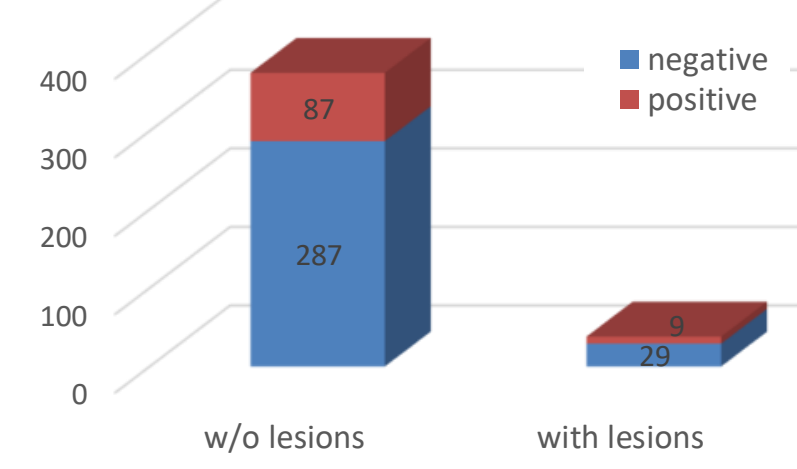

\section{Conclusions}

Hedgehogs admitted to rehabilitation centres are frequently contaminated by the zoonotic agent T. erinacei even in the absence of cutaneous lesions. Our results underline the importance of good management practices, especially with young animals, which can be hosted for long periods and may be grouped during their rehabilitation. They also alert about the possibility of animals being refractory to a treatment protocol including both topical and systemic antifungal drugs. 